\title{
Evaluation of the Aurora Kinase Inhibitor, ZM447439, in Canine Malignant Lymphoid Cells in Vitro
}

\author{
Keijiro Shiomitsu ${ }^{1}$, Xueqing Xia ${ }^{2}$, Kyle Waite ${ }^{1}$, Inder Sehgal ${ }^{2}$, Shulin $\mathrm{Li}^{2}$ \\ ${ }^{1}$ Cancer Treatment Unit, Department of Veterinary Clinical Sciences, Louisiana State University, Baton Rouge, USA \\ ${ }^{2}$ Department of Comparative Biomedical Sciences, Louisiana State University, Baton Rouge, USA \\ Email: keijiros@1su.edu, kshiomitsu@gmail.com
}

Received December 7, 2012; revised January 8, 2013; accepted February 1, 2013

\begin{abstract}
Aurora kinases play an important role in the cell cycle. These enzymes help establish mitotic spindles by directing centrosome duplication and separation and by regulating the spindle assembly checkpoint thereby helping control cytokinesis. An over-expression of aurora kinases has been reported in a variety of human tumors. In this study, we identified the expression of aurora-A and aurora-B kinases in canine malignant lymphoid cells. We also evaluated the effects of the aurora kinase inhibitor (ZM447439), and found that this inhibitor decreases cell viability, increases DNA content change, and leads to apoptosis in canine B- and T-cell lymphoid cell lines. The lymphotoxicity induced by ZM447439 in these canine lymphoid cell lines suggests that further in vivo evaluation of aurora kinase inhibitors as a potential treatment for canine malignant lymphoid tumors is warranted.
\end{abstract}

Keywords: Aurora Kinase Inhibitor; Canine Malignant Lymphoid Cells; Cell Cycle; Apoptosis; ZM447439

\section{Introduction}

Canine lymphoma, consisting of $\mathrm{B}$ and $\mathrm{T}$ cell lymphoma, is a very heterogeneous disease with both aggressive and indolent subtypes. Indolent subtypes of lymphoma have been reported in up to $29 \%$ of dogs [1]. In general, complete treatment response is rarely achieved with the indolent subtypes, but longer survival time has been observed [1]. Canine diffuse large B-cell lymphoma is typically an aggressive and high grade tumor in humans and dogs. The most common protocols for B-cell lymphoma are CHOP-based, which consist of cyclophosphamide, doxorubicin, vincristine and prednisone. Complete response rates range from approximately $80 \%-90 \%$, and median survival times range from approximately $12-14$ months with standard CHOP-based protocols [2,3]. T-cell lymphoma has a typically negative prognosis. MOPP protocols (Mechlorethamine, vincristine, prednisone, and procarbazine) for T-cell lymphoma have been reported to produce 189 days of progression-free survival [4]. However, even with high initial response rates with the CHOP based protocol, the majority of canine lymphoma patients die within 14 - 16 months.

The cell cycle is a highly regulated and complex process and is precisely controlled by numerous enzymes [5]. Disruption of this regulation sometimes results in unrestrained cell growth, a defining characteristic of all malignant tumors. Thus, inhibiting key enzymes which promote unchecked cell growth may prove to be a valu- able mode of cancer treatment. One of the critical enzyme families involved in the regulation of the cell cycle are the aurora kinases.

Aurora kinases are a family of serine/threonine kinases that are evolutionarily highly conserved. The most important function of the aurora kinases is to promote mitotic spindle assembly by regulating centrosome duplication and separation [6]. Aurora kinases are composed of three family members: aurora-A, aurora-B and aurora-C. Aurora-A and aurora-B have been extensively studied, and the function of these kinases is well understood, but little is known about aurora-C [7]. Previous studies have shown that aurora-A and aurora-B are overexpressed by a variety of tumors, including bladder, breast, colorectal, esophageal, glioma, pancreatic and prostate tumors in humans [8-13]. Aurora-B is located at chromosome $17 \mathrm{p}$ 13.1, and localizes at centromeres during prometaphase; then relocates to the central spindle and the midbody during anaphase and telophase $[14,15]$. Aurora- $\mathrm{B}$ is also a part of the chromosome passenger complex, which contains important factors such as the inner centromere proteins borealin and survivin, necessary for proper kinetochore-microtubule attachment [16].

ZM447439(N-[4-[[6-Methoxy-7-[3-(4-morpholinyl)pr-opoxy]-4-quinazolinyl]amino]phenyl]benzamide) is a 513.6 Dalton ATP-competitive selective inhibitor of aurora-A and aurora-B, which disturbs chromosomal alignment and cell division, as well as mitotic checkpoints in 
Hela, A549, MCF-7, and DLD1 cells [17]. Reduction of phosphorylated Histone $\mathrm{H} 3$ also occurs by inhibiting aurora-A and aurora-B [17]. ZM447439 does not interfere with the activities of regular cell cycles in normal cells, and does not affect important regulators such as cdc2, cdc25, MAPK (Mitogen-Activated Protein Kinase) or cyclin B [18]. Aurora kinases are now an attractive target in human cancer therapy, and intensive research including preclinical and clinical trials have been reported [19-23]; however, to our knowledge, scant information exists on the efficacy of aurora kinase inhibition in veterinary medicine.

In this study, we hypothesize that inhibiting aurora kinase will increase DNA content, and lead to the death of canine malignant lymphoid cells in vitro. The objectives of this study were to identify the expression of aurora kinases in canine B- and T-lymphoid cell lines; to evaluate the in vitro cytotoxic effect of an aurora kinase inhibitor on these tumor cells; to evaluate the effect of aurora kinase inhibition on cell cycle kinetics; and to determine the mechanism of cellular death in the canine malignant lymphoid cell lines.

\section{Materials and Methods}

\subsection{Cell Culture}

Two anaplastic canine lymphoid cell lines (GL-1 and EMA), Canine Aortic Endothelial Cells (CnAOEC), and the human pancreatic carcinoma cell line (PANC-1) were used. The canine lymphoid cell lines were kindly donated by Dr. Munekazu Nakaichi and Dr. Takuya Mizuno from Yamaguchi University, Yamaguchi city (Japan). CnAOEC and PANC-1 were purchased from Cell Applications Inc. (San Diego, CA) and the American Type Culture Collection (ATCC, Manassas, VA) respectively. GL1 has a B-cell and EMA has a T-cell immunophenotype. The characteristics of the lymphoid cell lines used in this study have been partially described $[24,25]$. GL-1 and EMA, were maintained in RPMI-1640 (ATCC, Manassas, VA) supplemented with $10 \%$ fetal bovine serum (FBS, purchased from the ATCC, Manassas, VA) and $2 \mathrm{mM}$ L-glutamine (ATCC, Manassas, VA). CnAOEC was maintained in Canine Endothelial Cell Growth Medium (Cell Application Inc., San Diego, CA). PANC-1 was maintained in Dulbecco's Modified Eagle's Medium (DMEM, ATCC, Manassas, VA) supplemented with 10\% FBS. All cell cultures were maintained in $5 \% \mathrm{CO}_{2}$ at $37^{\circ} \mathrm{C}$ in a humidified incubator, and the same conditions were used for the MTT assay, cell-cycle analysis, and apoptosis assay.

\subsection{Drug}

ZM447439 (aurora kinase-B inhibitor: Tocris Bioscience, Ellisville, MO) was purchased as a powder and stored at $-20^{\circ} \mathrm{C}$, until the preparation of a stock solution. ZM447439 stock solutions $(10 \mathrm{mM})$ were prepared in dimethylsulfoxide (DMSO), and stored at $-20^{\circ} \mathrm{C}$, until use.

\subsection{RNA Preparation}

Total RNA was isolated from GL-1, EMA, and CnAOEC cells, using TRIzol Reagent (Invitrogen, Carlsbad, CA). Approximately $5 \times 10^{6}$ cells were used. The RNA concentration was determined by NanoDrop ${ }^{\circledR}$ ND-1000 (Wilmington, DE). Approximately $1.0 \mu \mathrm{g} / \mu \mathrm{l}$ of RNA was acquired by the kit. Residual genomic DNA was removed from total RNA using DNase I (Applied Biosystems, Carlsbad, CA). Two micrograms of RNA were used for cDNA synthesis using the High Capacity RNA-to-cDNA Kit (Applied Biosystems, Carlsbad, CA). The following conditions were used for cDNA synthesis: $25^{\circ} \mathrm{C}$ for 10 minutes, $37^{\circ} \mathrm{C}$ for 2 hours followed by enzyme inactivation at $85^{\circ} \mathrm{C}$ for 5 minutes using the GeneAmp ${ }^{\circledR}$ PCR System 9700 ( Applied Biosystem, Carlsbad, CA).

\subsection{RT-PCR}

Aurora-A and aurora-B cDNA sequences, from human (GeneBank accession: NM_198437.1 and, NM_004217.2, respectively) and cow (GeneBank Accession: NM_00103 8028.1 and NM_183084.2), were aligned and consensus primers (Table $\overline{\mathbf{1}}$ ) were designed using the Primer 3 program (http://frodo.wi.mit.edu/primer3/). Canine specific glyceraldehyde-3-phosphate dehydrogenase (GAPDH, GeneBank Accession: NM 001003142.1) primers were also designed in the same manner. The PCR cycling conditions were as follows: $5 \mathrm{~min}$ at $95^{\circ} \mathrm{C}$, followed by 30 cycles of $40 \mathrm{~s}$ at $95^{\circ} \mathrm{C}$ (denaturing), $40 \mathrm{~s}$ at $57^{\circ} \mathrm{C}$ (annealing), and $45 \mathrm{~s}$ at $72^{\circ} \mathrm{C}$ (extension). PCR amplification of aurora-A, aurora-B, and GAPDH, was performed using $0.5 \mu \mathrm{l}$ of cDNA, $2 \mu \mathrm{l}$ of forward and reverse primers, $20.5 \mu \mathrm{l}$ of PCR quality sterile water, and $25 \mu \mathrm{l}$ of PCR Master mix (Qiagen Inc., Valencia, CA) in a total volume of $50 \mu \mathrm{l}$. The primer sets and the size of target amplicons are shown in Table 1. Each primer set was designed to amplify partial sequences, not full-length. The PCR products were separated by electrophoresis at $90 \mathrm{~V}$ for 30 minutes on $2 \%$ agarose gels with $0.01 \%$ of ethidium bromide, and then those were analyzed by FluorChem ${ }^{\circledR}$ Q (Alpha Innotech, Santa Clara, CA) under UV light. The target PCR products of aurora-A and aurora-B were sequenced with the 3130 Genetic Analyzer (Applied Biosystems, Carlsbad, CA).

\subsection{Western Blot}

Five $\times 10^{6}$ GL-1, EMA, CnAOEC, and PANC-1 cells were harvested from T-25 flasks, and lysed with RIPA buffer (Santa Cruz Biotechnology, Santa Cruz, CA) ac- 
Table 1. Primers and PCR amplicon sizes in RT-PCR.

\begin{tabular}{cllc}
\hline Target & Direction & Primer Sequence (5'-3') & Amplicon Size (bp) \\
\hline Aurora-A & Forward & CAGCCATAAACCGGCTCAGA & 661 \\
& Reverse & TCTCCTGGAGGATGGAGCAT & 428 \\
Aurora-B & Forward & CCTCATGGAGCCGCTCCAAT & 499 \\
& Reverse & CCTCCATGATCGTGGCTGTT & \\
FAPDH & Forward & TCCATCTTCCAGGAGCGAGA & ATACATTGGGGGTGGGGACA \\
\hline
\end{tabular}

cording to the manufacturer's protocol. Protein concentration was determined by the BCA protein assay according to the manufacturer's protocol (Bio-Rad, Hercules, CA). Western blot analysis was performed with $10 \%$ SDS-PAGE. Protein $(10 \mu \mathrm{g})$ was mixed with a standard gel loading buffer. After boiling for 5 minutes, the samples were loaded in each lane. The SDS-PAGE gel was transferred to nitrocellulose membranes (0.45 um Pro$\tan ^{\circledR}$, Whatman International Ltd., Maidstone, Kent, UK) by electroblotting at $90 \mathrm{~V}$ for 1.5 hour with standard transfer buffer. Blots were blocked overnight at $4{ }^{\circ} \mathrm{C}$ with $5 \%$ non-fat dry milk in phosphate-buffered saline (PBS) containing $0.2 \%$ TWEEN-20. The blots were probed with anti-rabbit Glyceraldehyde 3-phosphate dehydrogenase (GAPDH; 1:2000 dilution; monoclonal mouse antibody, Abcam, Cambridge, MA), anti-human aurora-A (1:1000 dilution; polyclonal goat antibody, Tocris Bioscience, Ellisville, MO), and anti-human aurora-B (1:1000 dilution; polyclonal rabbit antibody, Abcam, Cambridge, MA) antibodies at room temperature for 1 hour. Between blocking and antibody incubations, the membranes were washed with PBS containing 0.2\% TWEEN-20. Prior to development, membranes were then incubated with an appropriate horseradish peroxidase conjugated secondary antibody (1:2000 dilution; Santa Cruz Biotechnology, Santa Cruz, CA) at room temperature for 1 hour. The proteins were visualized using Amersham ECL plus substrate (GE Healthcare, Buckinghamshire).

\subsection{MTT Assay}

To evaluate the effect of ZM447439 on cell viability, MTT assays (Roche Applied Science, Indianapolis, IN) were performed following the manufacturer's instructions. Briefly, approximately $3 \times 10^{5} \mathrm{GL}-1$ and $6 \times 10^{5}$ EMA cells were placed in 96-well plates. Due to the different cell growth rates, different cell numbers were used. The plated cells were counted manually by hemocytometer. Various concentrations $(0.25,0.5,2.5,5,10,20,40$ $\mu \mathrm{M}$ with GL-1 and 1, 2.5, 5, 10, 20, 40, $80 \mu \mathrm{M}$ with EMA) of ZM447439 were placed in each well for 48 hours. Ten microliters of MTT labeling reagent was applied to each well for 4 hours, and then one hundred microliters of solubilization solution were added. The plates were incubated overnight. The absorbance of the samples at $570 \mathrm{~nm}$ was then measured by a standard microplate reader. Cell viability was calculated as a percentage of the absorbance of the treated cells relative to the absorbance of the control cells (cell viability $\%=$ absorbance of treated cells/absorbance of control). Non-treated cells were incubated with vehicle $(0.5 \%$ of DMSO), and served as controls. Treated cells were exposed to both ZM447439 and DMSO since DMSO is the solvent. Untreated cells were exposed to the high concentration of DMSO $(0.5 \%)$ and compare to the treated cells to control for the effect of DMSO on cell viability. Dose response curves were generated, and the $\mathrm{IC}_{50}$ was calculated for GL-1 and EMA. The samples were run in duplicate and the experiments were repeated at least three times.

\subsection{Cell Cycle Analysis}

To assess the effect of aurora kinase inhibitors on the cell cycle, cell cycle analysis was performed. The cell cycle analysis was performed with propidium iodide (SigmaAldrich, St. Louis, MO) staining of DNA with the BD FACSCalibur (BD Biosciences, San Jose, CA). Approximately $1 \times 10^{6}$ GL-1 and EMA cells were cultured in 48-well plates. ZM447439 used at its $\mathrm{IC}_{50}$ concentration was placed in each well for 24 or 48 hours. The treated cells were washed twice with PBS, and then resuspended in $100 \mu \mathrm{l}$ of PBS. Ice-cold methanol was added slowly, and incubated at $4^{\circ} \mathrm{C}$ for 30 minutes, to fix the cells. The cells were then washed with PBS, and then resuspended in $450 \mu \mathrm{l}$ of PBS, $25 \mu \mathrm{l}$ of RNAse A (Sigma-Aldrich, St. Louis, MO) and $25 \mu$ l of propidium iodide and incubated for 30 minutes at room temperature in the dark. The stained cells were gated and 30,000 events were counted by flow cytometry. The data was analyzed and reported as a percentage of each cell-cycle phase, which are G1, S and $\mathrm{G} 2 / \mathrm{M}$, and the DNA content $(2 \mathrm{~N}, 4 \mathrm{~N}$, and $8 \mathrm{~N})$ was indicated. Untreated cells served as control. This control was not treated with $0.5 \%$ DMSO. The samples were run in duplicate, and the experiments were repeated at least three 
times.

\subsection{Apoptosis Assay}

To determine a possible mechanism of cell death caused by the ZM447439, the Annexin V apoptosis assay (BD Biosciences, San Jose, CA) was performed following the manufacturer's instructions.

For the Annexin V assay, approximately $1 \times 10^{6} \mathrm{GL}-1$ and EMA cells were cultured in 48 -well plates. The $\mathrm{IC}_{50}$ of ZM447439 was placed in each well for 24 or 48 hours. The treated cells were washed twice with cold PBS, and then resuspended in $100 \mu \mathrm{l}$ of binding buffer. Then, $5 \mu \mathrm{l}$ of Annexin $\mathrm{V}$ and $5 \mu$ of propidium iodide (PI) were added, and incubated for 15 minutes at room temperature in the dark. An additional $400 \mu \mathrm{l}$ of binding buffer was added to each tube, and then analyzed by flow cytometry within 1 hour. Dual staining of Annexin V and PI is considered late apoptosis, and Annexin V staining without PI staining is considered early apoptosis. PI staining alone was considered necrosis. Untreated cells served as a control. This control was not treated with $0.5 \%$ DMSO. The samples were run in duplicate and the experiments were repeated at least three times.

\subsection{Statistical Analysis}

All data was analyzed using either Graph Pad Prism version 5 (Graph Pad Prism Software Inc., La Jolla, CA) or SAS version 9.3 (SAS Institute Inc., Cary, NC). Graph Pad Prism was used to calculate the $\mathrm{IC}_{50}$ for each cell line used in the MTT assay and generate graphs. To summarize the data, the mean and standard deviation (SD) were calculated for the MTT, cell cycle assay and Annexin $\mathrm{V}$ assays. For the cell cycle assay, an ANOVA with a factorial arrangement of treatments (DNA content, incubation period, and interaction of DNA content and incubation period) was used. When overall significance was found, post hoc comparisons were conducted with Tukey's test for main effects and with pairwise tests of least squares means for interaction effects. To assess each group for the Annexin V assay, an ANOVA was performed, and then the post hoc Tukey's test was used for pairwise comparisons of significant overall effects. A p value $<0.05$ was considered significant.

\section{Results}

\subsection{Expression of Aurora Kinase in Malignant Lymphoid Cells}

\subsubsection{RT-PCR}

The CnAOEC cells served as a positive control. To confirm the expression of aurora kinase-A and -B in canine malignant lymphoid cell lines and in CnAOEC cells, RT-PCR was performed, (Figure 1) and then the PCR products of aurora-A and aurora-B were sequenced (Figure 2). The canine aurora-A showed a $90 \%$ homology to human aurora-A and the canine aurora-B showed a $92 \%$ homology to human aurora-B. The sequences of aurora-A and aurora-B were deposited in GeneBank (Aurora-A; KC127668, Aurora-B; KC137547).

\subsubsection{Protein Expression of Aurora Kinases}

To detect aurora-A and aurora-B kinase in normal canine cells, as well as malignant human and canine cells, western blot analysis was performed on protein extracts from PANC-1, CnAOEC, and the canine malignant lymphoid cell lines GL-1 and EMA. Aurora-A and aurora-B kinases were detected in PANC-1, CnAOEC, and canine malignant lymphoid cell lines. The expression of aurora-A in both canine lymphoid cell lines, GL-1 and EMA, appeared more intense than in CnAOEC cells but less than in PANC-1 cells. Alternatively, the expression of aurora-B in GL-1 and EMA cell lines appeared much more intense than in both the PANC-1 and CnAOEC cells (Figure 3) indicating that this aurora kinase was relatively selective for canine lymph malignancy. The intensity of expression of aurora-A and aurora-B for each cell line in the western blots was consistent with the results of RT-PCR.

\subsubsection{Cell Viability with Various Concentrations of ZM447439 in GL-1 and EMA Cell Lines}

Cell survival was assessed after 24 hours of incubation with ZM 447439 in GL-1 and EMA lymphoid cell lines (Figure 4). Dose response curves were generated for GL-1 and EMA, and the $\mathrm{IC}_{50}$ was calculated as 4.77 and $8.03 \mu \mathrm{M}$, respectively. These $\mathrm{IC}_{50}$ levels in $\mathrm{GL}-1$ and EMA were used for subsequent experiments with the ZM 447439 inhibitor. A dose-dependent cytotoxicity and cell growth inhibition were observed in both GL-1 and EMA cells treated with ZM 447439.

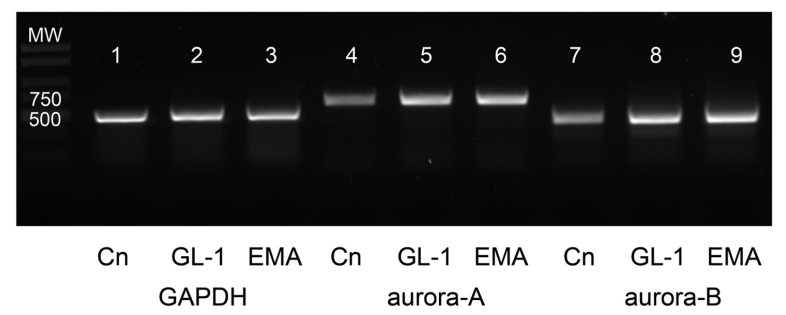

Figure 1. Results of RT-PCR of GAPDH, aurora-A and aurora-B from CnAOEC, GL-1 and EMA cell lines. Amplicons in lanes 1, 2, 3 are GAPDH, amplicons in lanes 4, 5, 6 are aurora-A, and amplicons in lanes $7,8,9$ are aurora-B. CnAOEC and GAPDH serve as positive controls. Expression of GAPDH is identical in all samples. Clear bands of aurora-A and aurora-B were detected from CnAOEC, GL1 and EMA. Decreased expression of aurora-A and aurora$B$ were noted in CnAOEC compared to GL-1 and EMA. Cn represents canine aortic endothelial cells. 


\subsubsection{DNA Content Change with ZM447439 in GL-1 and EMA}

The cellular DNA component was assessed by flow cytometry after 24 and 48 hours of incubation with the $\mathrm{IC}_{50}$ of ZM447439 in GL-1 and EMA cells (Figures 5(A)-(F)). The DNA content of the GL-1 and EMA cells was present in the diploid $(2 \mathrm{~N})$, tetraploid $(4 \mathrm{~N})$ and octoploid $(8 \mathrm{~N})$ states. The representative numeric results are shown as the mean \pm standard deviation (SD). The portion of the $4 \mathrm{~N}$ and $8 \mathrm{~N}$ cells increased after 24 and 48 hours of incubation with ZM447439 in both GL-1 and EMA cells (Figures 5(G) and $(\mathbf{H}), \mathrm{p}<0.05)$. Details of the mean value of the $2 \mathrm{~N}, 4 \mathrm{~N}$ and $8 \mathrm{~N}$ states for the control after 24 and 48 hours of incubation are shown in Table 2 . The portion of the $2 \mathrm{~N}$ DNA content in GL-1 cells decreased over the 48 hours of incubation. The portion of the $2 \mathrm{~N}$ DNA in EMA cells, however, was found to increase after 48 hours of incubation. The details of the mean value of the cell cycle analysis in GL-1 and EMA cells are shown as the mean \pm SD (Figures 5(G) and (H), Table 2).

\subsubsection{Induced Apoptosis with ZM447439 in GL-1 and EMA Cells}

Annexin V Assay. To determine the mechanism of cell death with ZM447439, an Annexin V assay was performed. Flow cytometric analysis was performed after 24 and 48 hours of incubation with the $\mathrm{IC}_{50}$ of ZM447439 in GL-1 and EMA cells (Figures 6(A)-(F)). The Annexin $\mathrm{V}^{+} / \mathrm{PI}^{-}$population represents early apoptosis, the Annexin $\mathrm{V}^{+} / \mathrm{PI}^{+}$population represents late apoptosis, and PI staining alone represents necrosis. A significant increase of early apoptosis was seen in EMA cells at 24 hours, and both GL-1 and EMA cells at 48 hours (Figures 6(G) and (H), $\mathrm{p}<0.05)$. A significant increase of late apoptosis was also seen in both GL-1 and EMA cells at 24 and 48 hours, but the fraction of cells in late apoptosis was

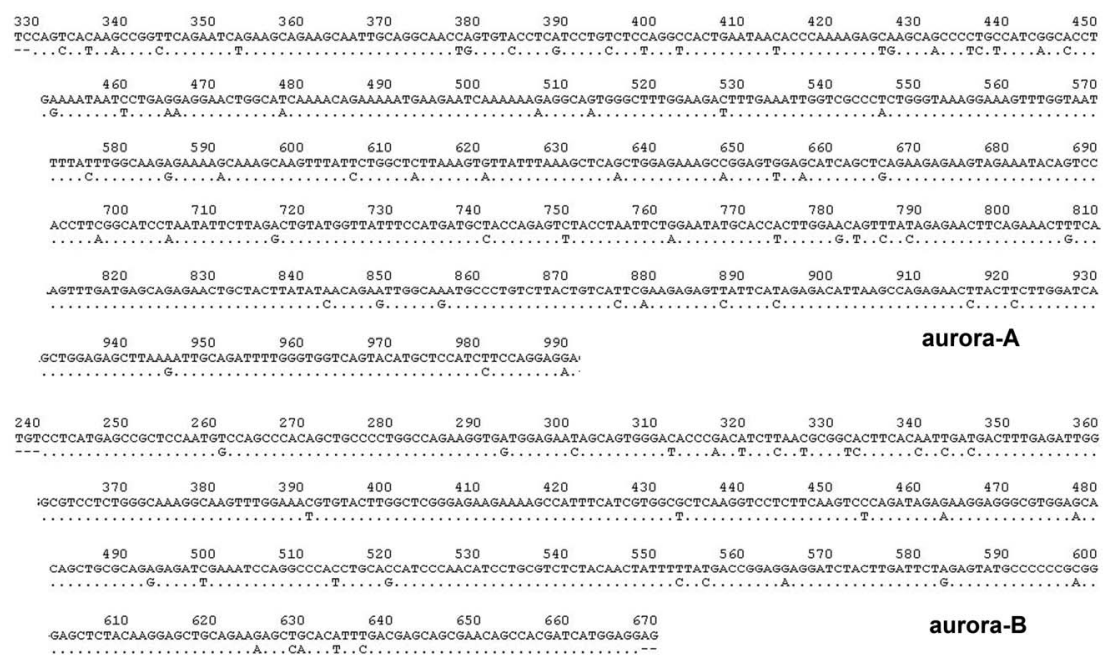

Figure 2. Sequence alignment results between human and canine aurora-A and aurora-B. The canine aurora-A showed a $\mathbf{9 0 \%}$ homology to human aurora-A, and the canine aurora-B showed a $92 \%$ homology to human aurora-B. Human sequences are located on the top and canine sequences are located on the bottom for both aurora-A and aurora-B. Conserved nucleotide residues (A), (G), (C) and (T)) are represented as dots, and each number indicates the location of the nucleotide residues from the beginning of each sequence.

Table 2. The numeric cell cycle and apoptosis assay results of the impact of ZM447439 on GL-1 and EMA cells. The representative numeric results are shown as the mean $(\%) \pm$ standard deviation (SD). Tables $A$ and $B$ are the results of the cell cycle analysis, and Tables $C$ and $D$ are the results of the apoptosis assays. The incubation period with ZM447439 ranges from 0 (control) to 48 hours. EA represents early apoptosis and LA represents late apoptosis.

\begin{tabular}{|c|c|c|c|c|c|c|c|}
\hline A & & & & B & & & \\
\hline GL-1 & $2 \mathrm{~N}$ & $4 \mathrm{~N}$ & $8 \mathrm{~N}$ & EMA & $2 \mathrm{~N}$ & $4 \mathrm{~N}$ & $8 \mathrm{~N}$ \\
\hline Control & $98.4 \% \pm 0.6 \%$ & $1.5 \% \pm 0.6 \%$ & $0 \%$ & Control & $95.3 \% \pm 2.0 \%$ & $4.6 \% \pm 2.0 \%$ & $0 \%$ \\
\hline 24 hours & $19.4 \% \pm 7.9 \%$ & $74.3 \% \pm 2.7 \%$ & $6.1 \% \pm 5.9 \%$ & 24 hours & $37.5 \% \pm 1.4 \%$ & $50.6 \% \pm 8.1 \%$ & $7.2 \% \pm 2.1 \%$ \\
\hline 48 hours & $6.0 \% \pm 1.5 \%$ & $61.4 \% \pm 5.3 \%$ & $29.4 \% \pm 5.0 \%$ & 48 hours & $56.4 \% \pm 8.9 \%$ & $22.8 \% \pm 3.4 \%$ & $16.7 \% \pm 10.0 \%$ \\
\hline $\mathrm{C}$ & & & & $\mathrm{D}$ & & & \\
\hline GL-1 & EA & LA & & EMA & EA & LA & \\
\hline 24 hours & $8.2 \% \pm 0.9 \%$ & $6.2 \% \pm 2.5 \%$ & & 24 hours & $28.9 \% \pm 3.9 \%$ & $20.9 \% \pm 3.1 \%$ & \\
\hline 48 hours & $16.0 \% \pm 4.6 \%$ & $8.7 \% \pm 2.1 \%$ & & 48 hours & $38.6 \% \pm 9.1 \%$ & $39.9 \% \pm 9.6 \%$ & \\
\hline
\end{tabular}




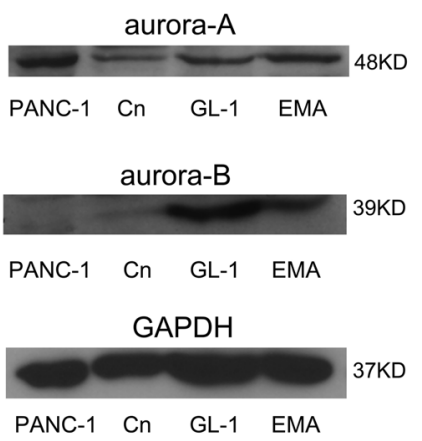

Figure 3. Western blot analysis. Proteins from the PANC-1, CnAOEC, GL-1 and EMA cells were extracted and subjected to western blot analysis. The membrane was probed with anti-aurora-A, anti- aurora-B and anti-GAPDH. PANC1, CnAOEC, and GAPDH served as controls. The band density of GAPDH is relatively identical through all samples. Cn represents canine aortic endothelial cells.
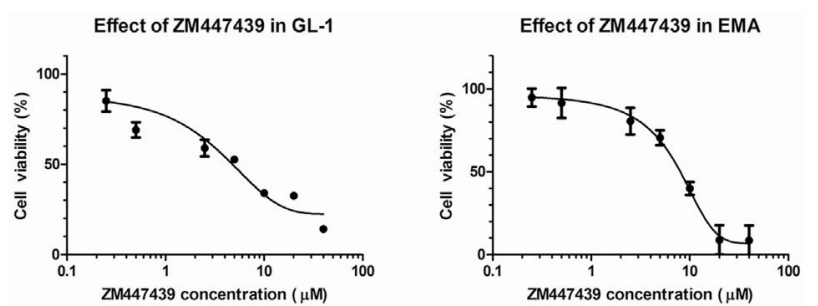

Figure 4. Cell viability of two canine lymphoid cell lines (GL-1 and EMA). GL-1 and EMA cells were cultured for 24 hours with various concentrations of $Z$ M447439. The mean of the 3 experiments \pm SD cell viability was measured by MTT assay. The absorbance was measured at $570 \mathrm{~nm}$ and the cell viability was calculated as the mean absorbance of the treated cells divided by the mean absorbance of the control. The $\mathrm{IC}_{50}$ of the GL-1 and EMA cells was calculated as 4.77 and $8.03 \mu \mathrm{M}$, respectively. The samples were run in duplicate and the experiments were repeated three times.

greater in EMA cells than in GL-1 cells. The details of the mean value of early and late apoptosis after 24 and 48 hours of incubation are shown as the mean \pm SD for the control, GL-1 and EMA cells (Figures 6(G) and (H), Table 2).

\section{Discussion}

Aurora kinases are a very attractive target for antitumor therapies because of their central role in the regulation of the cell cycle. A number of small-molecule aurora kinase inhibitors have recently been developed and intensively investigated both experimentally and in numerous clinical trials in human medicine [16,21-23]. However, there are no published data showing the effect of aurora kinase inhibitors in veterinary medicine at the time of this writing. In the present study, we evaluated the effect of ZM447439 in the canine lymphoid cell lines GL-1 and
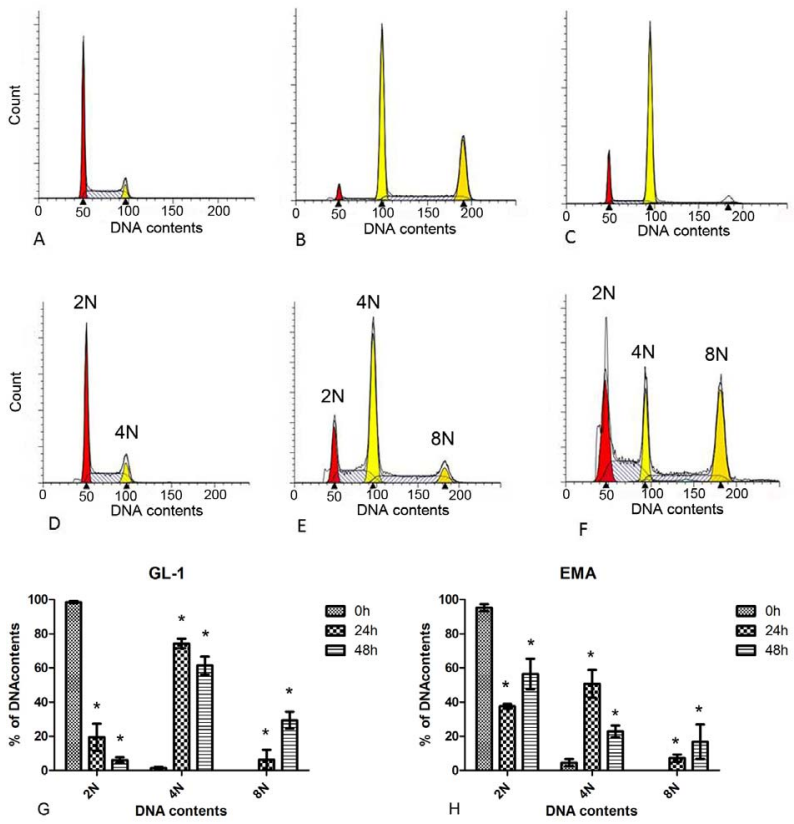

Figure 5. The cell cycle effect of ZM447439 in GL-1 and EMA cells. This figure shows the DNA content change in GL-1 (A), (B), and (C) and EMA (D), (E), and (F) cells. Figures $A$ and $D$ are controls. Figures $B$ and $E$ show results at 24 hours, and Figures $C$ and $F$ show results at 48 hours after incubation with the $\mathrm{IC}_{50}$ of $\mathrm{ZM447439}$. The DNA component was assessed by flow cytometry after 24 and 48 hours of incubation with ZM447439. $2 \mathrm{~N}, 4 \mathrm{~N}$, and $8 \mathrm{~N}$ indicate DNA content. The results shown are the mean values of the 3 experiments \pm SD (G) and (H). The samples were run in duplicate and the experiments were repeated at least three times. "Values are significantly different $(p<0.05)$ for each control.

EMA. In general, malignant lymphoid diseases, such as lymphoma and leukemia, have a short doubling time, which is consistent with a high rate of mitosis, indicating the involvement of aurora kinases. No published data existed regarding the expression of aurora kinase-A and/or -B in dogs, so we began by investigating their expression in normal canine cells. Both aurora kinase-A and aurora kinase-B were successfully detected by RTPCR and western blot in a normal canine cell line (Figures 1 and 3). Sequence alignment between human and canine aurora-A and aurora-B was performed, and it revealed that aurora-A has a $90 \%$ homology to human aurora- $\mathrm{A}$, and the canine aurora-B has a $92 \%$ homology to human aurora-B. This homology of aurora-A and auroraB suggests highly conserved genes between humans and canine species. RT-PCR band intensities of aurora-A and aurora-B were more intense in two malignant canine lymphoid cell lines than in normal canine endothelial cells, which is consistent with an increased mRNA expression of these aurora kinases in malignancy. However, we did not quantitate the mRNA expression levels of aurora-A 

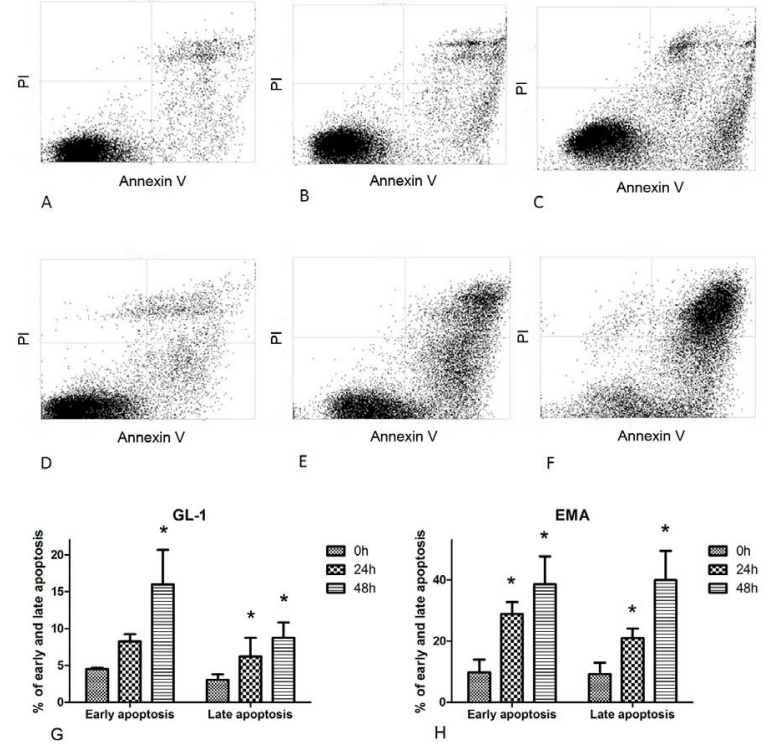

Figure 6. The impact of ZM447439 on GL-1 (A)-(C) and EMA (D)-(F)) cells. This figure shows early apoptosis (Annexin $\mathrm{V}^{+}, \mathrm{PI}^{-}$) and late apoptosis (Annexin $\mathrm{V}^{+}, \mathrm{PI}^{+}$), as detected by Annexin $V$ and PI staining. Figures $A$ and $D$ are controls. Figures $B$ and $E$ show results 24 hours, and $C$ and $F$ show results 48 hours after incubation with the $\mathrm{IC}_{50}$ of ZM447439. The controls and treated samples were stained with Annexin V and PI, and then analyzed by flow cytometry. An increased population of Annexin V and PI stained cells were found in both GL-1 and EMA cells after 24 and 48 hours of incubation with the IC50 of ZM447439. The results shown are the mean values of the 3 experiments \pm SD ( $G$ and $H)$. The samples were run in duplicate and the experiments were repeated at least three times. "Values are significantly different $(p<0.05)$ for each control.

and -B, and quantitative RT-PCR will be required to better understand the RNA level expression of aurora kinases in canine malignant lymphoid cells.

Protein expression of aurora-A and aurora-B was also evaluated by western blot. More intense bands of aurora$\mathrm{A}$ and aurora-B were noted in the canine malignant lymphoid cell lines, which is a finding similar to the RT-PCR results. The aurora-B expression seems to appear greater than aurora-A in the lymphoid cell lines and aurora-B was more selectively expressed than aurora-A in the malignant cells than in the CnAOEC. These data suggest that aurora-B might be a more important target in canine malignant lymphoid disease. There is evidence in the human tumor literature for such tumor-type predominance of one aurora kinase sub-type. For example, Eiseman et al. have reported that $83 \%$ of anaplastic human thyroid cancer cases express aurora-A, but only $13 \%$ of the cases express aurora-B [26]. Other investigators have shown that over-expression of aurora kinases might be correlated with tumor recurrence, grades, and/or survival [27-29]. Since mitotic figure frequency is one of the im- portant prognostic factors in histological grades, future investigations are necessary to correlate the level of aurora kinase expression with mitotic figures and prognosis in canine mast cell tumors and/or soft tissue sarcomas. Other groups have suggested that over-expression seems not to reflect just malignancy but also tumor grade [30]. In general, the malignant tumor cell lines should have unique characteristics since those cell lines are selected and become immortal during the establishment of the cell lines. Although we have shown possible overexpression of aurora-A and aurora-B in established malignant lymphoid cell lines, this finding must next be validated in clinical tissues.

We have shown that ZM447439 inhibits cell growth and decreases cell viability in a dose dependent manner. This observation is very similar to previously reported studies using human malignant cell lines [31,32]. When the $\mathrm{IC}_{50}$ of the ZM447439 was compared in GL-1 and EMA cells, GL-1 (B-cell) was more susceptible than EMA (T-cell) (the $\mathrm{IC}_{50}$ of GL-1 and EMA was calculated as 4.77 and $8.03 \mu \mathrm{M}$, respectively), which might correlate with drug resistance. It is well known that T-cell lymphomas are more drug resistant than B-cell lymphomas, and usually T-cell disease also has a poorer prognosis [33]. Since observed drug susceptibility might be correlated with drug resistance, it is possible that p-glycoprotein or other drug transporter protein expression differs between GL-1 and EMA cells.

Cell cycle analysis was performed to determine the effect of the ZM447439 on the cell cycles of GL-1 and EMA cells. Increased tetraploidy and octoploidy (4N and $8 \mathrm{~N}$ content) were seen after 24 and 48 hours of incubation with ZM447439 in both the GL-1 and EMA cells. These results indicate that cell division is prevented by ZM447439. Keen et al. have suggested calling aurora kinases "mitotic drivers" instead of mitotic inhibitors since these drugs interfere with proper cell division rather than interfering with movement into the $\mathrm{M}$ phase. We observed a more pronounced cell distribution change in 2N DNA in GL-1 cells than EMA cells.

In the present study, we also have shown that cell death was induced by ZM447439 in both canine malignnant lymphoid cell lines. Annexin V staining indicated significant increases of early apoptosis in EMA cells at 24 hours, and late apoptosis in both GL-1 and EMA cells after 48 hours incubation with ZM447439 ( $p<0.05)$. Early and late apoptosis were more obvious in EMA than GL-1, which suggests that a cytotoxic effect is dominant in EMA while a cytostatic effect is dominant in GL-1 with a lower concentration of ZM447439. The response of EMA cells to ZM447439 observed in this study is similar to observations in human aurora kinase studies, but the different forms of responses (cytotoxic vs. cytostatic) are not typically seen in human aurora kinase studies. In general, a cytotoxic effect is the typical observa- 
tion. However, in mouse model studies, it has been observed that tumor growth remains stable after the initiation of aurora kinases, which suggests a cytostatic effect by a variety of aurora kinases [34,35]. The different tumor responses to aurora kinase inhibition might correlate with clinical response and prognosis, and will be an important subject to assess in clinical trials.

Our results, although promising, must be interpreted with caution. All data in the present study were obtained in vitro and must now be validated in vivo or in a clinical setting. Secondly, the $\mathrm{IC}_{50}$ reported here is higher than previously reported in human cell line studies. Most of the previous reports have shown an $\mathrm{IC}_{50}$ in nano-molar ranges, not micro-molar ranges [30]. To achieve proper drug blood concentration levels without toxicity, it would be essential to evaluate the normal tolerable toxicity with a mouse model before performing studies with canine patients. Adequate cytotoxic/cytostatic effects might be achieved with plasma drug concentrations below the $\mathrm{IC}_{50}$ in vivo due to the humoral immune response, which means higher doses may not be necessary in a clinical setting. Thirdly, quantitative gene expression determination of aurora kinases with qRT-PCR would be a necessary step to understand better tumor behaviors such as progression, drug response, and prognosis in canine malignant lymphoid cells. Since our present study indicates that ZM447439 was selectively toxic to canine lymphoid tumor cells, further preclinical studies are warranted. These would include in vivo tumor suppression assays in nude mice, oral pharmacokinetics and toxicity testing.

In conclusion, we have detected the protein expression of aurora kinase-A and aurora kinase-B in a normal canine cell line and malignant lymphoid cells. Tetraploid and octoploid DNA was seen 24 and 48 hours after incubation with ZM447439. This suggests substantial interference with tumor cell division. ZM447439 induced apoptotic cell death particularly in EMA cells. These results indicate that aurora kinase inhibitors could be a very attractive therapeutic agent for malignant canine lymphoid tumors, and provide justification for further in vivo study.

\section{Acknowledgements}

This study was supported by the Canine Health Foundation. The authors would like to thank Marilyn Dietrich, and In Joong Kim for technical help. The authors would like to thank Michel Kearney for the statistical analysis. The authors also would like to thank Dr. Munekazu Nakaichi and Dr. Takuya Mizuno (Yamaguchi University, Japan) for kindly donating the lymphoid cell lines.

\section{REFERENCES}

[1] K. E. Flood-Knapik, A. C. Durham, T. P. Gregor, M. D.
Sánchez, M. E. Durney and K. U. Sorenmo, "Clinical, Histopathological and Immunohistochemical Characterization of Canine Indolent Lymphoma," Veterinary Comparative Oncology, 2012, in press.

[2] L. D. Garrett, D. H. Thamm, R. Chun, R. Dudley and D. M. Vail, "Evaluation of 6-Month Chemotherapy Protocol with No Maintenance Therapy for Dogs with Lymphoma," Journal of Veterinary Internal Medicine, Vol. 16, No. 6, 2002, pp. 704-709.

[3] R. Chun, L. D. Garrentt and D. M. Vail, "Cancer Chemotherapy," In: S. J. Withrow and D. M. Vail, Eds., Withrow and MacEwen's Small Animal Clinical Oncology, 4th Edition, Saunders Elsevier, St. Louis, 2007, pp. 163-191.

[4] E. M. Brodsky, G. N. Maudlin, J. L. Lachowicz and G. S. Post, "Asparaginase and MOPP Treatment of Dogs with Lymphoma," Journal of Veterinary Internal Medicine, Vol. 23, No. 3, 2009, pp. 578-584. doi:10.1111/j.1939-1676.2009.0289.x

[5] K. A. Schafer, "The Cell Cycle: A Review," Veterinary Pathology, Vol. 35, No. 6, 1998, pp. 461-478. doi: $10.1177 / 030098589803500601$

[6] G. Mountzios, E. Terpos and M. A. Dimopoulos, "Aurora Kinases as Targets for Cancer Therapy," Cancer Treatment Review, Vol. 34, No. 2, 2008, pp. 175-182. doi:10.1016/j.ctrv.2007.09.005

[7] S. S. Farag, "The Potential Role of Aurora Kinase Inhibitors in Haematological Malignancies," British Journal of Haematology, Vol. 155, No. 5, 2011, pp. 561-579. doi:10.1111/j.1365-2141.2011.08898.x

[8] S. Sen, H. Zhou, R. D. Zhang, D. S. Yoon, F. VakarLopez, S. Ito, F. Jiang, D. Johnston, H. B. Grossman, A. C. Ruifrok, R. L. Katz, W. Brinkley and B. Czerniak, "Amplification/Overexpression of a Mitotic Kinase Gene in Human Bladder Cancer," Journal of the National Cancer Institute, Vol. 94, No. 17, 2002, pp. 1320-1329. doi:10.1093/jnci/94.17.1320

[9] T. Takahashi, M. Futamura, N. Yoshimi, J. Sano, M. Katada, Y. Takagi, M. Kimura, T. Yoshioka, Y. Okano, and S. Saji, "Centrosomal Kinase, HsAIRK1 and HsAIRK3, Are Overexpressed in Primary Colorectal Cancers," Japanese Journal of Cancer Research, Vol. 91, No. 10, 2000, pp. 1007-1014. doi:10.1111/j.1349-7006.2000.tb00878.x

[10] T. Tong, Y. Zhong, J. Kong, L. Dong, Y. Song, M. Fu, Z. Liu, M. Wang, L. Guo, S. Lu, M. Wu and Q. Zhan, "Overexpression of Aurora-A Contributes to Malignant Development of Human Esophageal Squamous Cell Carcinoma," Clinical Cancer Research, Vol. 10, No. 21, 2004, pp. 7304-7310. doi:10.1158/1078-0432.CCR-04-0806

[11] A. Klein, W. Reichardt, V. Jung, K. D. Zang, E. Meese and S. Urbschat, "Overexpression and Amplification of STK15 in Human Gliomas," International Journal of Oncology, Vol. 25, No. 6, 2004, pp. 1789-1794.

[12] S. Rojanala, H. Han, R. M. Muńoz, W. Browne, R. Nagle, D. D. Von Hoff and D. J. Bearss, "The Mitotic Serine Threonine Kinase, Aurora-2, Is a Potential Target for Drug Development in Human Pancreatic Cancer," Molecular Cancer Therapeutics, Vol. 3, No. 4, 2004, pp. 451457.

[13] H. M. Buschhorn, R. R. Klein, S. M. Chambers, M. C. 
Hardy, S. Green, D. Bearss and R. B. Nagle, "Aurora-A Overexpression in High-Grade PIN Lesions and Prostate Cancer," Prostate, Vol. 64, No. 4, 2005, pp. 341-346. doi:10.1002/pros.20247

[14] M. Carmena and W. C. Earnshaw, "The Cellular Geography of Aurora Kinases," Nature Reviews. Molecular Cell Biology, Vol. 4, No. 11, 2003, pp. 842-845. doi: $10.1038 / \mathrm{nrm} 1245$

[15] M. T. Parra, A. Viera, R. Gómez, J. Page, M. Camena, W. C. Earnshaw, J. S. Rufas and J. A. Suja, "Dynamic Relocalization of the Chromosomal Passenger Complex Proteins Inner Centromere Protein (INCENP) and Aurora-B Kinase during Male Mouse Meiosis," Journal of Cell Science, Vol. 116, No. 6, 2003, pp. 961-974. doi: $10.1242 /$ jes. 00330

[16] J. J. Kitzen, M. J. de Jonge and J. Verweij, “Aurora Kinase Inhibitors," Critical Reviews in Oncology/Hematology, Vol. 73, No. 2, 2010, pp. 99-110. doi:10.1016/j.critrevonc.2009.03.009

[17] C. Ditchfield, V. L. Johnson, A. Tighe, R. Ellston, C. Haworth, T. Johnson, A. Mortlock, N. Keen and S. S. Taylor, "Aurora B Couples Chromosome Alignment with Anaphase by Targeting BubR1, Mad2, and Cenp-E to Kinetochores, "Journal Cell Biology, Vol. 161, No. 2, 2003, pp. 267-280. doi:10.1083/jcb.200208091

[18] B. B. Gadea and J. V. Ruderman, "Aurora Kinase Inhibitor ZM447439 Blocks Chromosome-induced Spindle Assembly, the Completion of Chromosome Condensation, and the Establishment of the Spindle Integrity Checkpoint in Xenopus Egg Extracts," Molecular Biology of the Cell, Vol. 16, No. 3, 2005, pp. 1305-1318. doi:10.1091/mbc.E04-10-0891

[19] N. Steeghs, F. A. Eskens, H. Gelderblom, J. Verweij, J. W. Nortier, J. Ouwerkerk, C. van Noort, M. Mariani, R. Spinelli, P. Carpinelli, B. Laffranchi and M. J. de Jonge, "Phase I Pharmacokinetic and Pharmacodynamic Study of the Aurora Kinase Inhibitor Danusertib in Patients with Advanced or Metastatic Solid Tumors," Journal of Clinical Oncology, Vol. 27, No. 30, 2009, pp. 5094-5101. doi:10.1200/JCO.2008.21.6655

[20] T. Macarulla, A. Cervantes, E. Elez, E. Rodriguez-Braun, J. Baselga, S. Roselló, G. Sala, I. Blasco, H. Danaee, Y. Lee, J. Ecsedy, V. Shinde, A. Chakravarty, D. Bowman, H. Liu, O. Eton, H. Fingert and J. Tabernero, "Phase I Study of the Selective Aurora A Kinase Inhibitor MLN8054 in Patients with Advanced Solid Tumors: Safety, Pharmacokinetics, and Pharmacodynamics," Molecular Cancer Therapeutics, Vol. 9, No. 10, 2010, pp. 28442852. doi:10.1158/1535-7163.MCT-10-0299

[21] A. M. Traynor, M. Hewitt, G. Liu, K. T. Flaherty, J. Clark, S. J. Freedman, B. B. Scott, A. M. Leighton, P. A. Watson, B. Zhao, P. J. O'Dwyer and G. Wilding, "Phase I Dose Escalation Study of MK-0457, a Novel Aurora Kinase Inhibitor, in Adult Patients with Advanced Solid Tumors," Cancer Chemotherapy and Pharmacology, Vol. 67, No. 2, 2011, pp. 305-314. doi:10.1007/s00280-010-1318-9

[22] J. R. Diamond, B. R. Bastos, R. J. Hansen, D. L. Gustafson, S. G. Eckhardt, E. L. Kwak, S. S. Pandya, G. C. Fletcher, T. M. Pitts, G. N. Kulikowski, M. Morrow, J.
Arnott, M. R. Bray, C. Sidor, W. Messersmith and G. I. Shapiro, "Phase I Safety, Pharmacokinetic, and Pharmacodynamics Study of ENMD-2076, a Novel Angiogenic and Aurora Kinase Inhibitor, in Patients with Advanced Solid Tumors," Clinical Cancer Research, Vol. 17, No. 4, 2011, pp. 849-860. doi:10.1158/1078-0432.CCR-10-2144

[23] A. Chakravarty, V. Shinde, J. Tabernero, A. Ceravantes, R. B. Cohen, E. C. Dees, H. Burris, J. R. Infante, T. Macarulla, E. Elez, J. Andreu, E. Rodriguez-Braun, S. Rosello, M. von Mehren, N. J. Meropol, C. J. Langer, B. ONeil, D. Bowman, M. Zhang, H. Danaee, L. FaronYowe, G. Gray, H. Liu, J. Pappas, L. Silverman, C. Simpson, B. Stringer, S. Tirrell, O. P. Veiby, K. Venkatakrishnan, K. Galvin, M. Manfredi and J. A. Ecsedy, "Phase I Assessment of New Mechanism-Based Pharmacodynamics Biomarkers for MLN8054, a Small-Molecule Inhibitor of Aurora a Kinase," Cancer Research, Vol. 71, No. 3, 2011, pp. 675-685. doi:10.1158/0008-5472.CAN-10-1030

[24] M. Nakaichi, Y. Taura, M. Kanki, K. Mamba, Y. Momoi, H. Tsujimoto and A. Nakama, "Establishment and Characterization of a New Canine B-Cell Leukemia Cell Line," Journal of Veterinary Medical Science, Vol. 58, No. 5, 1996, pp. 469-471. doi:10.1292/jvms.58.469

[25] H. Hiraoka, K. Minami, N. Kaneko, T. Shimokawa Miyama, Y. Okamura, T. Mizuno and M. Okuda, “Aberrations of the FHIT Gene and Fhit Protein in Canine Lymphoma Cell Lines," Journal of Veterinary Medical Science, Vol. 71, No. 6, 2009, pp. 769-777. doi:10.1292/jvms.71.769

[26] S. M. Wiseman, H. Masoudi, P. Niblock, D. Turbin, A. Rajput, J. Hay, S. Bugis, D. Filipenko, D. Huntsman and B. Gilks, "Anaplastic Thyroid Carcinoma: Expression Profile of Targets for Therapy Offers New Insights for Disease Treatment," Annals of Surgical Oncology, Vol. 14, No. 2, 2007, pp. 719-729. doi:10.1245/s10434-006-9178-6

[27] Z. Guan, X. R. Wang, X. F. Zhu, X. F. Huang, J. Xu, L. H. Wang, X. B. Wan, Z. J. Long, J. N. Liu, G. K. Feng, W. Huang, Y. X. Zeng, F. J. Chen and Q. Liu, “Aurora-A, a Negative Prognostic Marker, Increases Migration and Decreases Radiosensitivity in Cancer Cells," Cancer Research, Vol. 67, No. 21, 2007, pp. 10436-10444. doi:10.1158/0008-5472.CAN-07-1379

[28] R. Reiter, P. Gais, U. Jütting, M. K. Steuer-Vogt, A. Pickhard, K. Bink, S. Rauser, S. Lassmann, H. Höfler, M. Werner and A. Walch, "Aurora Kinase A Messenger RNA Overexpression Is Correlated with Tumor Progression and Shortened Survival in Head and Neck Squamous Cell Carcinoma," Clinical Cancer Research, Vol. 12, No. 17, 2006, pp. 5136-5141. doi:10.1158/1078-0432.CCR-05-1650

[29] F. Yang, X. Guo, D. G. Rosen and J. Liu, “AURKA and BRCA2 Expression Highly Correlate with Prognosis of Endometrioid Ovarian Carcinoma," Modern Pathology, Vol. 24, No. 6, 2011, pp. 836-845.

doi:10.1038/modpathol.2011.44

[30] N. Mori, C. Ishikawa, M. Senba, M. Kimura and Y. Okano, "Effects of AZD1152, a Selective Aurora B Kinase Inhibitor, on Burkitt's and Hodgkin's Lymphomas," Biochemical Pharmacology, Vol. 81, No. 9, 2011, pp. 1106- 
1115. doi:10.1016/j.bcp.2011.02.010

[31] T. Ikezoe, J. Yang, C. Nishioka, T. Tasaka, A. Taniguchi, Y. Kuwayama, N. Komatsu, K. Bandobashi, K. Togitani, H. P. Koeffler and H. Taguchi, "A Novel Treatment Strategy Targeting Aurora Kinases in Acute Myelogenous Leukemia," Molecular Cancer Therapeutics, Vol. 6, No. 6, 2007, pp. 1851-1857. doi:10.1158/1535-7163.MCT-07-0067

[32] I. Georgieva, D. Koychev, Y. Wang, J. Holstein, W. Hopfenmüller, M. Zeitz and P. Grabowski, "ZM447439, a Novel Promising Aurora Kinase Inhibitor, Provokes Antiproliferative and Proapoptotic Effects Alone and in Combination with Bio- and Chemotherapeutic Agents in Gastroenteropacreatic Neuroendocrine Tumor Cell Lines," Neuroendocrinology, Vol. 91, No. 2, 2010, pp. 121-130. doi:10.1159/000258705

[33] L. M. Beaver, G. Strotttner and M. K. Klein, "Response Rate after Administration of a Single Dose of Doxorubicin in Dogs with B-Cell or T-Cell Lymphoma: 41 Cases
(2006-2008)," Journal of American Veterinary Medical Association, Vol. 237, No. 9, 2010, pp. 1052-1055. doi:10.2460/javma.237.9.1052

[34] R. W. Wilkinson, R. Odedra, S. P. Heaton, S. R. Wedge, N. J. Keen, C. Crafter, J. R. Foster, M. C. Brady, A. Bigley, E. Brown, K. F. Byth, N. C. Barrass, K. E. Mundt, K. M. Foote, N. M. Heron, F. H. Jung, A. A. Mortlock, F. T. Boyle and S. Green, "AZD1152, a Selective Inhibitor of Aurora B Kinase, Inhibits Human Tumor Xenograft Growth by Inducing Apoptosis," Clinical Cancer Research, Vol. 13, No. 12, 2007, pp. 3682-3688. doi:10.1158/1078-0432.CCR-06-2979

[35] R. P. Evans, C. Naber, T. Steffler, T. Checkland, C. A. Maxwell, J. J. Keats, A. R. Belch, L. M. Pilarski, R. Lai and T. Reiman, "The Selective Aurora B Kinase Inhibitor AZD1152 Is a Potential New Treatment for Multiple Myeloma," British Journal of Haematology, Vol. 140, No. 3, pp. 295-302. doi:10.1111/j.1365-2141.2007.06913.x 